\title{
AD/RHIC/RD-23
}

\author{
RHIC PROJECT \\ Brookhaven National Laboratory
}

The Calculation of the Influence on Beam Parameters in RHIC and Beam Size in Transport Line due to Change of AGS Beam Parameters

Jianming Xu

October 1990 


\section{The Calculation of the Influence on Beam Parameters in RHIC and Beam Size in Transport Line due to Change of AGS Beam Parameters}

\section{Jianming Xu}

The influences of the change of the AGS beam parameters on the beam parameters at the RHIC injection point has been summarized in reference 1 . In that calculation, the beam transport line from AGS to RHIC is the original one. ${ }^{2}$ For the modified transport ${ }^{3}$ line this influence has been calculated and the results are summarized in this report. In this calculation not only the phase space dilution in RHIC caused by the AGS beam parameter change but also the effect on the beam size in the beam transport line from AGS to RHIC have been calculated.

In this report $\beta_{x o}, \alpha_{x o}, D_{x o}, D_{x o}^{\prime}, \beta_{y o}, \alpha_{y o}, D_{y o}$ and $D_{y o}^{\prime}$ are the Twiss parameters of the AGS ejected beam, $\beta_{x f}, \alpha_{x f}, D_{x f}, D_{x f}^{\prime}, \beta_{y f}, \alpha_{y f}, D_{y f}$ and $D_{x f}^{\prime}$ are the parameters at the RHIC injection point and suffix $N$ means nominal value. And the nominal values at RHIC injection point are: $\beta_{x f N}=9.086, \beta_{y f N}=49.806, \alpha_{x f N}=\alpha_{y f N}=0, D_{x f N}=-0.781$, $D_{x f N}^{\prime}=D_{y f N}^{\prime}=0, D_{y f N}=0$.

\section{PHASE SPACE DILUTION}

The phase space dilution factors $F_{x}$ and $F_{y}$ in the $x-x^{\prime}$ and $y-y^{\prime}$ planes due to mismatch between the emittance ellipse of the injected beam and the acceptance ellipse of RHIC at the injection point are expressed as follows:

$$
F=\frac{1}{2}\left[\left(\frac{\beta_{f N}}{\beta_{f}}+\frac{\beta_{f}}{\beta_{f N}}+\alpha_{f}^{2} \frac{\beta_{f N}}{\beta_{f}}\right)+\sqrt{\left(\frac{\beta_{f N}}{\beta_{f}}+\frac{\beta_{f}}{\beta_{f N}}+\alpha_{f}^{2} \frac{\beta_{f N}}{\beta_{f}}\right)^{2}-4}\right] .
$$

The phase space dilution factor caused by dispersion mismatch between the injected beam and the RHIC lattice is expressed as following:

$$
F=\left[1+\frac{|\delta|}{\sqrt{\epsilon}} \sqrt{\gamma_{f N} D^{2}+\beta_{f N} D^{\prime 2}}\right]^{2}
$$

where $D=D_{f N}-D_{f}$ and $D^{\prime}=D_{f N}^{\prime}-D_{f}^{\prime}, \delta=\Delta p / p, \epsilon$ is the beam emittance.

1 The Influence of the AGS Beam Parameters on the Beam Parameters at the RHIC Injection Point, RHIC-RD-14, Jianming Xu, January 1990.

2 Beam Transfer from AGS to RHIC, RHIC Technical Note No. 47, J. Claus and H. Foelsche, 1988.

3 The Improvement of the Vertical Dispersion Matching of the Beam Transport Line Between AGS and RHIC, AD/RHIC/RD-22, Jianming Xu, June 1990. 
The above expressions are valid for $\alpha_{f N}=0.0$ as at the RHIC injection point. The results are summarized in Figures 1 to 4 .

\section{BEAM SIZE AT SA}

In the modified lattice there is a low $\beta$ waist for the stripping foil SA where the nominal values of both $\beta_{x}$ and $\beta_{y}$ are 6.14 meters and $\alpha=0$. The change of the Twiss parameters of AGS beam will cause a change in the $\beta$ values at that point. The results are summarized in Figures 5 and 6. From Figure 5 one can find that $\beta_{x}$ at SA is more sensitive to the change of AGS beam parameters. So the beam size at SA can be used as a measure of the change of AGS beam parameter. By installing a profile monitor near SA to measure the beam cross section, one can tune the strengths of quadrupoles UQ1 to UQ5 until a round beam with proper size is gotten so as to match the AGS beam parameter variations.

\section{MAXIMUM BEAM SIZE IN BEAM TRANSPORT LINE}

In calculating the beam size the beam emittance is assumed to be $0.8 \mathrm{~mm} \mathrm{mrad}$ in both directions. The maximum half width of the beam in $\mathrm{U}$-line, $\mathrm{V}$-line, $\mathrm{W}$-line and Big-Bend at different AGS beam parameters are summarized in Figure 7 to Figure 10 respectively. The maximum half height in $U$ and $V$ lines are summarized in Figures 11 and 12 respectively. The maximum beam half height in the dipoles of U-line, W-line, BigBend and BMI are shown in Figures 13 to 16 . From these figures one can find that the maximum beam height in the dipoles is not sensitive to the AGS beam Twiss parameters and in most cases it is about $7 \mathrm{~mm}$ which is about half of the useful aperture of the dipoles in the vertical direction. Some curves in the above mentioned figures are not smooth this is because that the locations of the maximum beam size at different cases may not in the same element in the line.

\section{MAXIMUM BEAM SIZE IN INJECTION ELEMENTS}

The maximum half width and height of the beam in the injection elements SI0, SI1, SI2 and KI are summarized in Figures 17 to 20 and 21 to 24 respectively which should be considered in defining the aperture of these elements. Some of the curves are also not smooth because the locations of the maximum beam size are not at the same place for different AGS beam parameters. 
THE INJECTION POSITION ERRORS INDUCED BY

\section{AGS BEAM POSITION CHANGE}

The relations between the position error $x_{f}\left(y_{f}\right)$ and direction error $x_{f}^{\prime}\left(y_{f}^{\prime}\right)$ at the injection point of RHIC and the errors of the AGS ejected beam $x_{o}, x_{o}^{\prime}\left(y_{o}, y_{o}^{\prime}\right)$ are as follows

$$
\begin{aligned}
& x_{f}=0.14 x_{o}-4.85 x_{o}^{\prime} \\
& x_{f}^{\prime}=0.26 x_{o}-1.96 x_{o}^{\prime} \\
& y_{f}=0.45 y_{o}+15.5 y_{o}^{\prime} \\
& y_{f}^{\prime}=-0.07 y_{o}-0.25 y_{o}^{\prime}
\end{aligned}
$$

\section{THE EFFECT IN TRANSVERSE MOTION OF THE MOMENTUM CHANGE OF AGS BEAM}

The beam parameters at the injection point of RHIC are as follows if the momentum of AGS beam changes $10^{-3}$ and there is no sextupole component in the magnets of the transport line. In this table the F's are the phase space dilution factor due to mismatch of the corresponding beam parameters at the RHIC injection point.

Table 1

\begin{tabular}{|c|ccc|ccc|ccc|}
\hline$\frac{\Delta p}{p}$ & $\beta_{x f}$ & $\alpha_{x f}$ & $F_{x}$ & $D_{x f}$ & $D_{x f}^{\prime}$ & $F_{x}$ & $\beta_{y f}$ & $\alpha_{y f}$ & $F_{y}$ \\
\hline $10^{-3}$ & 9.061 & -0.01 & 1.01 & -0.781 & 0.005 & 1.03 & 48.588 & -0.018 & 1.03 \\
$-10^{-3}$ & 9.080 & -0.01 & 1.01 & -0.783 & 0.002 & 1.01 & 50.962 & 0.021 & 1.02 \\
\hline
\end{tabular}

If there is sextupole component in the magnetic field of the beam transport line, the chromatic effect will be larger. In this calculation in bending magnets the strength of the sextupole component at the location $5 \mathrm{~cm}$ away from the center line is supposed to be $2 \times 10^{-3}$ of the strength of dipole component and in quadrupoles the field gradient at the location $5 \mathrm{~cm}$ away from the center line due to sextupole component is $5 \times 10^{-3}$ of that of the quadrupole component. Under the above mentioned condition the beam parameters at the RHIC injection point are shown in the following table.

Table 2

\begin{tabular}{|c|ccc|ccc|ccc|}
\hline$\frac{\Delta p}{p}$ & $\beta_{x f}$ & $\alpha_{x f}$ & $F_{x}$ & $D_{x f}$ & $D_{x f}^{\prime}$ & $F_{x}$ & $\beta_{y f}$ & $\alpha_{y f}$ & $F_{y}$ \\
\hline $10^{-3}$ & 9.253 & -0.04 & 1.05 & -0.741 & 0.006 & 1.05 & 47.416 & -0.023 & 1.05 \\
$-10^{-3}$ & 8.94 & 0.46 & 1.05 & -0.818 & -0.006 & 1.05 & 52.015 & 0.033 & 1.05 \\
\hline
\end{tabular}

$D_{y f}, D_{y f}^{\prime}, x_{f}, x_{f}^{\prime}, y_{f}$ and $y_{f}^{\prime}$ are negligibly small.

If the relative strength of the sextupole components in the magnets is Gaussian distribution with RMS value of $2 \times 10^{-3}, 5 \times 10^{-3}$ in dipoles and quadrupoles respectively, for 
6 different seeds the maximum dilution factors are about the same as shown in the above table except that due to dispersion mismatch, for which the largest one is 1.08 .

With these sextupole components in the transport line magnets, the maximum half width and half height of the beam in $\mathrm{mm}$ is as following:

In the $\mathrm{x}$-direction:

Table 3

\begin{tabular}{|c|c|c|c|c|c|c|c|c|}
\hline$\frac{\Delta p}{p}$ & U-line & W-line & V-line & Big-Bend & SI0 & SI1 & SI2 & KI \\
\hline $10^{-3}$ & 9.6 & 6.8 & 8.0 & 5.2 & 5.5 & 6.7 & 7.0 & 3.2 \\
$-10^{-3}$ & 9.4 & 6.8 & 8.4 & 5.1 & 5.7 & 7.0 & 7.3 & 3.2 \\
0 & 9.1 & 6.6 & 8.1 & 5.1 & 5.6 & 6.9 & 7.1 & 3.2 \\
\hline
\end{tabular}

In the $\mathrm{y}$-direction

Table 4

\begin{tabular}{|c|c|c|c|c|c|c|c|c|c|}
\hline$\frac{\Delta p}{p}$ & $\begin{array}{c}\text { U-line } \\
\text { Dipole }\end{array}$ & $\begin{array}{c}\text { U-line } \\
\text { Quad }\end{array}$ & $\begin{array}{c}\text { W } \\
\text { line }\end{array}$ & $\begin{array}{c}\text { V } \\
\text { line }\end{array}$ & $\begin{array}{c}\text { Big } \\
\text { Bend }\end{array}$ & BMI & SI0/SI1 & SI2 & KI \\
\hline $10^{-3}$ & 6.1 & 8.6 & 6.6 & 9.5 & 5.8 & 6.1 & $6.2 / 6.2$ & 4.3 & 6.2 \\
$-10^{-3}$ & 6.0 & 8.7 & 6.8 & 9.6 & 5.9 & 6 & $6.3 / 6.3$ & 4.4 & 6.3 \\
0 & 6.1 & 8.5 & 6.6 & 9.6 & 5.6 & 5.7 & $6.2 / 6.2$ & 4.3 & 6.2 \\
\hline
\end{tabular}

From Tables 3 and 4, it is clear that, the beam cross section is not sensitive to beam energy change. But one should pay attention to the central orbit change due to dispersion effect and the beam envelop will be larger than the values shown in Table 3 . In $y$-direction, the dispersion function is small, its maximum value is only 0.36 meters and only introduces $0.36 \mathrm{~mm}$ central orbit shift for $\frac{\Delta p}{p}=10^{-3}$. So that the beam envelop is about the same values as shown in Table 4. The maximum central orbit shift in $x$-direction and the maximum $x$-coordinates of beam envelop are shown in the following table.

\begin{tabular}{|c|c|c|c|c|c|c|c|c|}
\hline Lines & U-line & W-line & V-line & Big-Bend & SI0 & SI1 & SI2 & KI \\
\hline$x_{c, \max }$ & 4.5 & 3.1 & $\sim 0$ & 2.2 & 0.5 & 1 & 1.1 & 0.9 \\
$x_{\max }$ & 13.8 & 9.9 & 8.4 & 7.3 & 6.2 & 8 & 8.4 & 4.1 \\
\hline
\end{tabular}




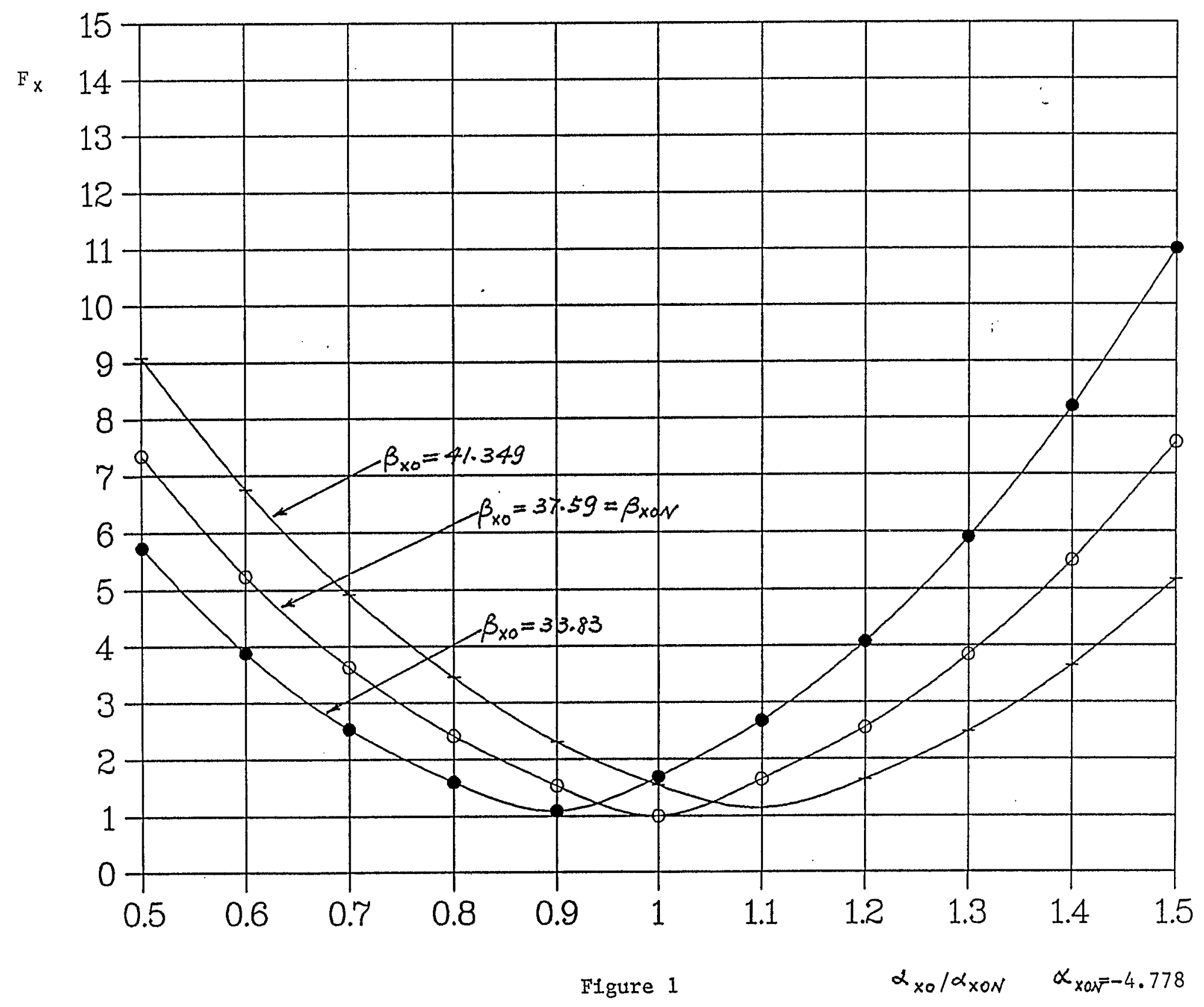




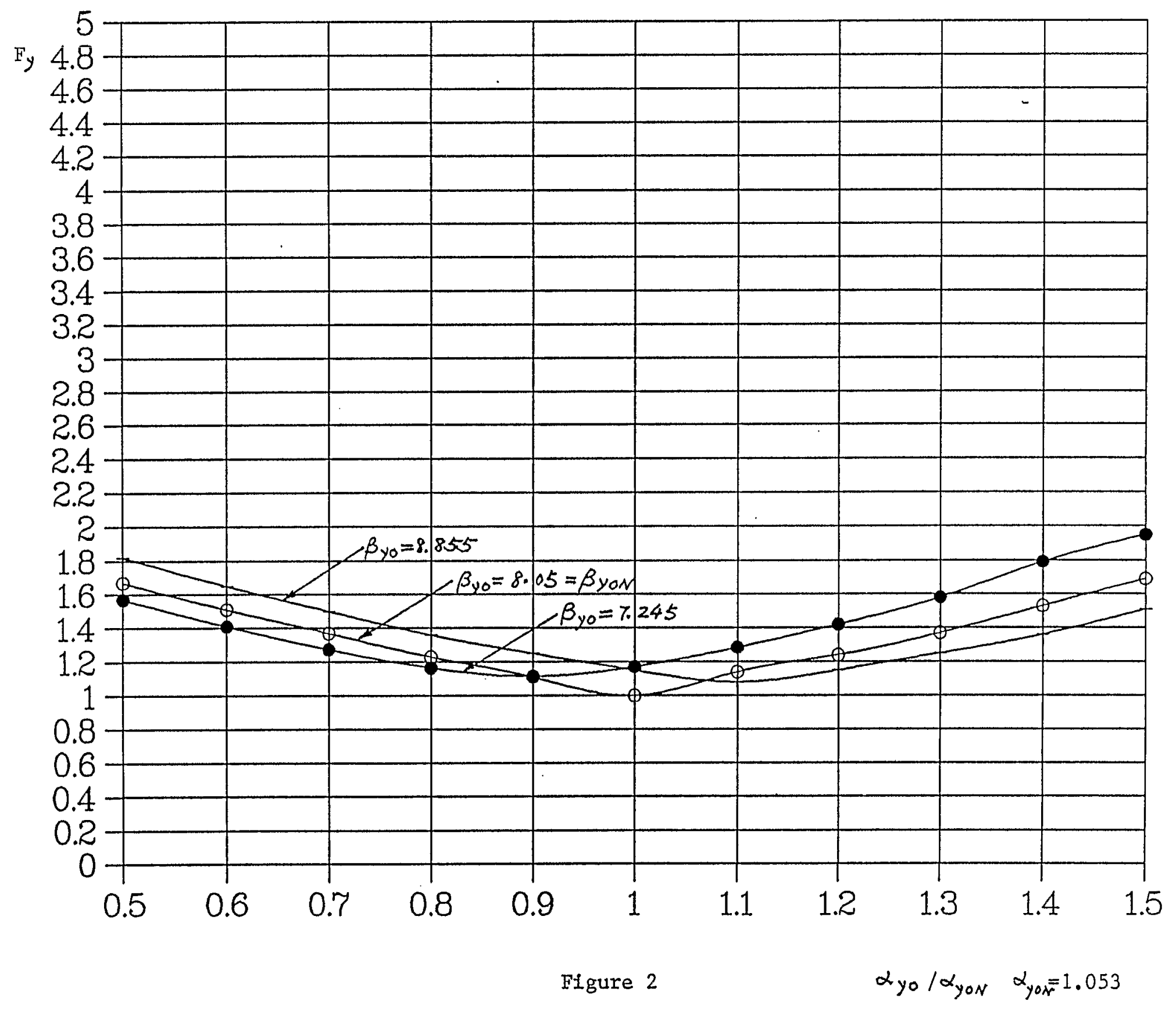




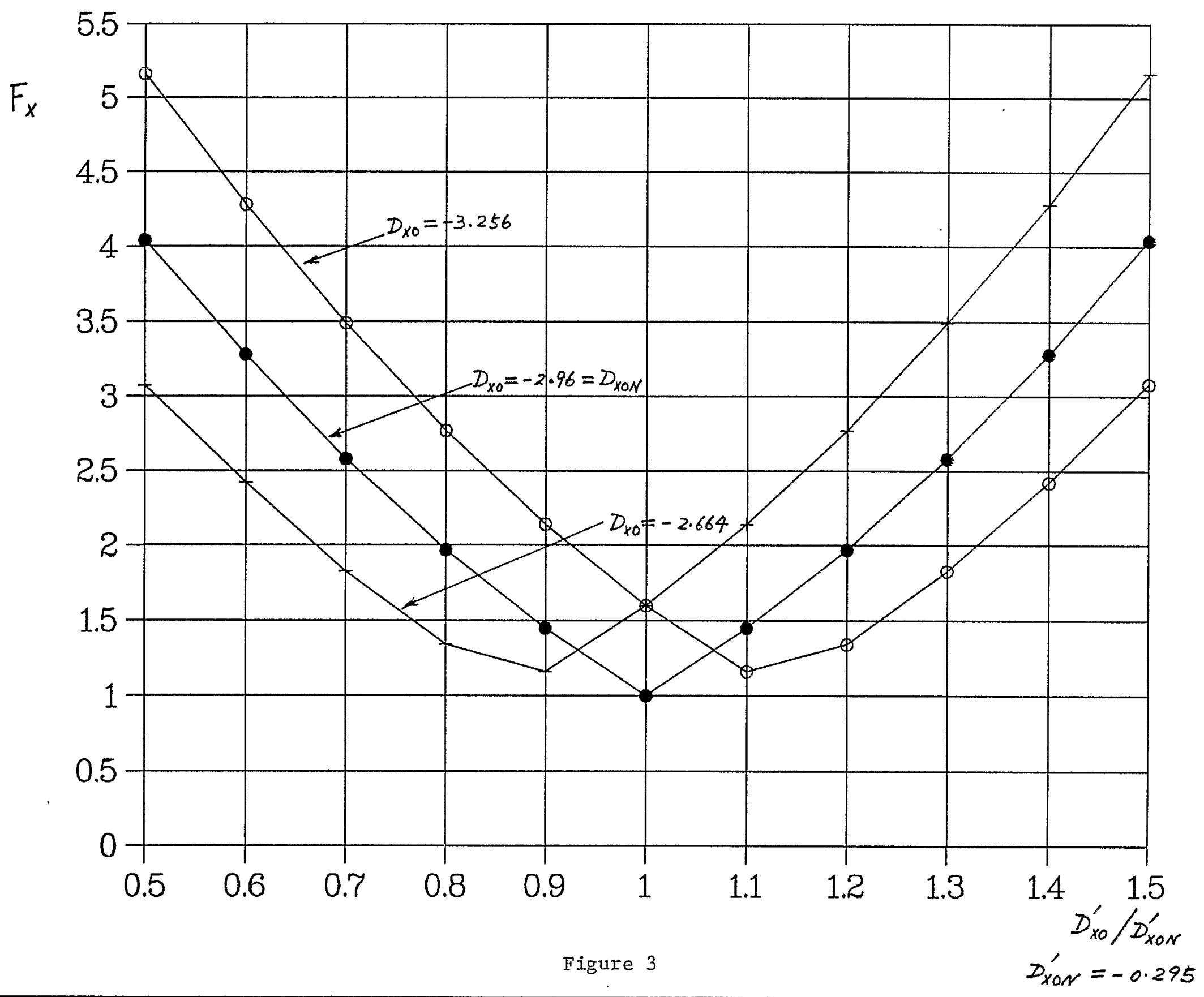


Fy

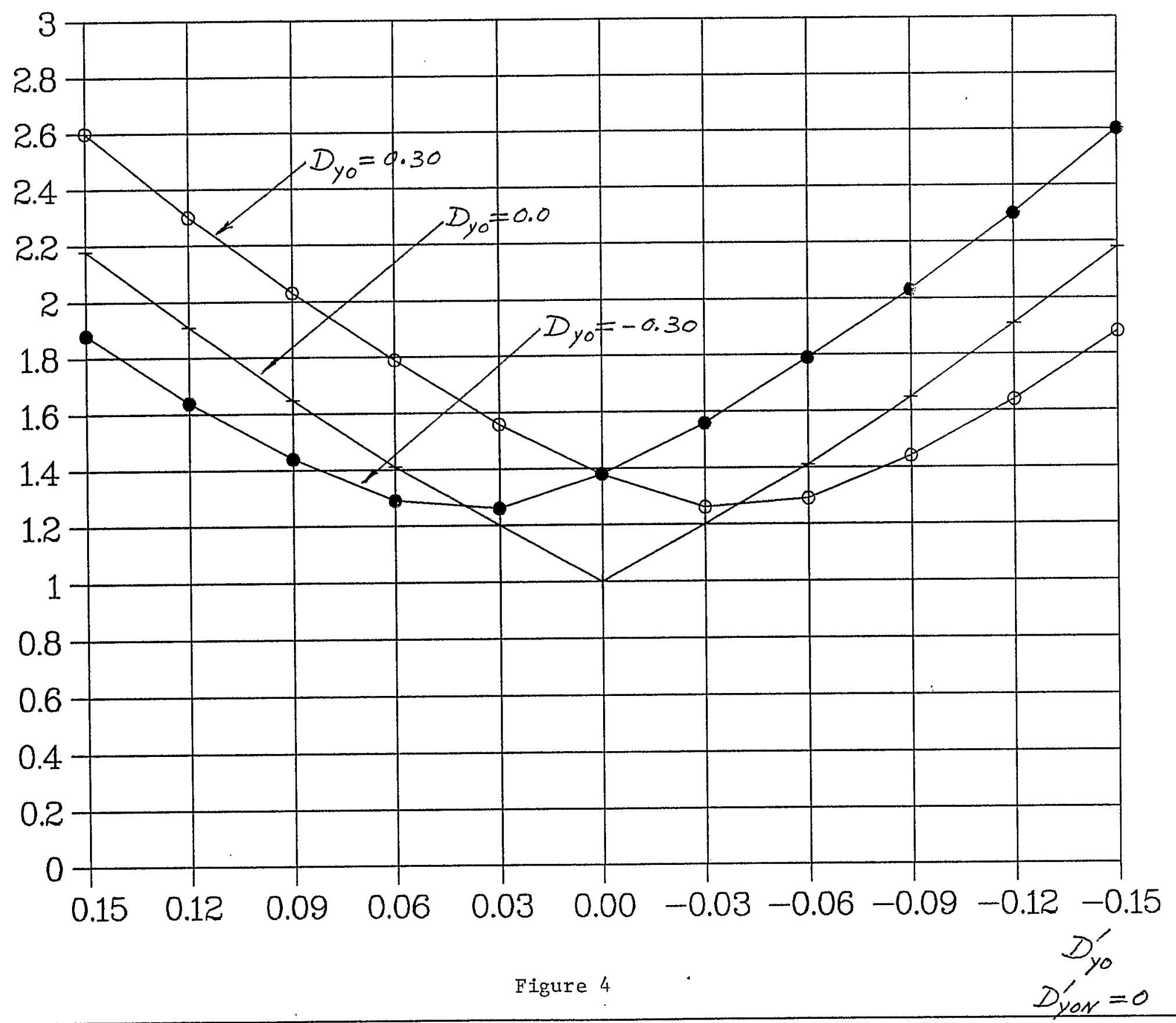




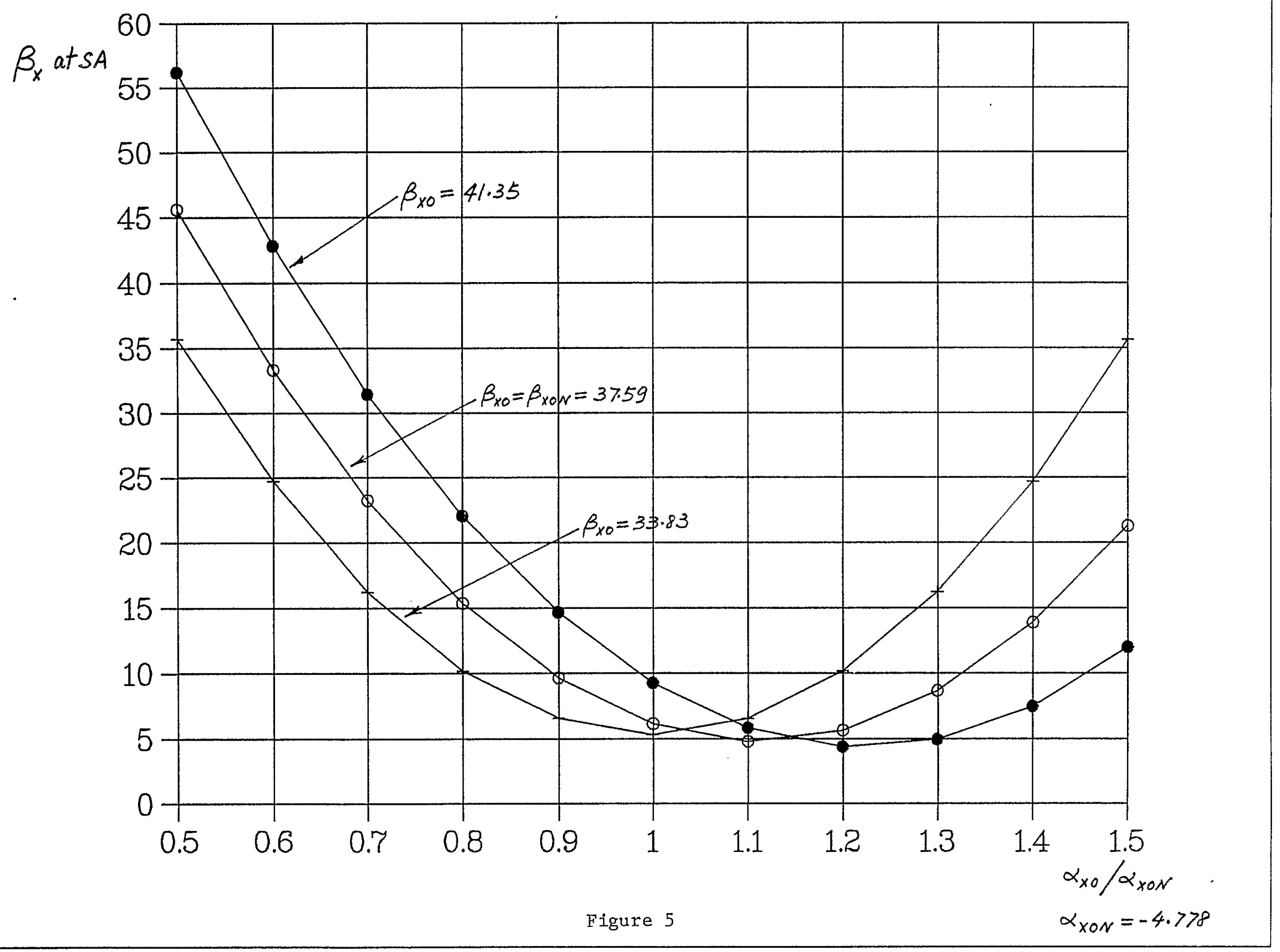




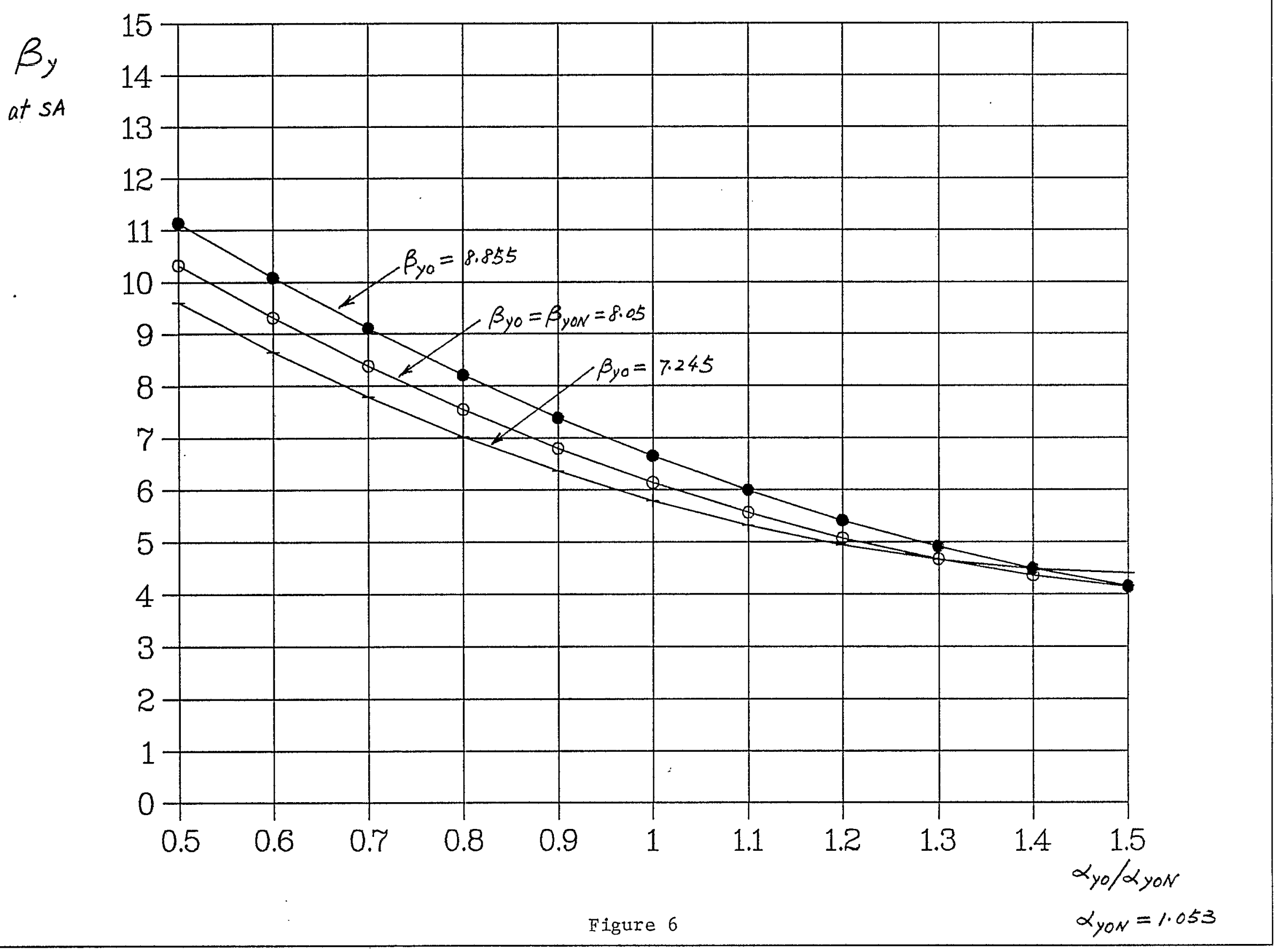




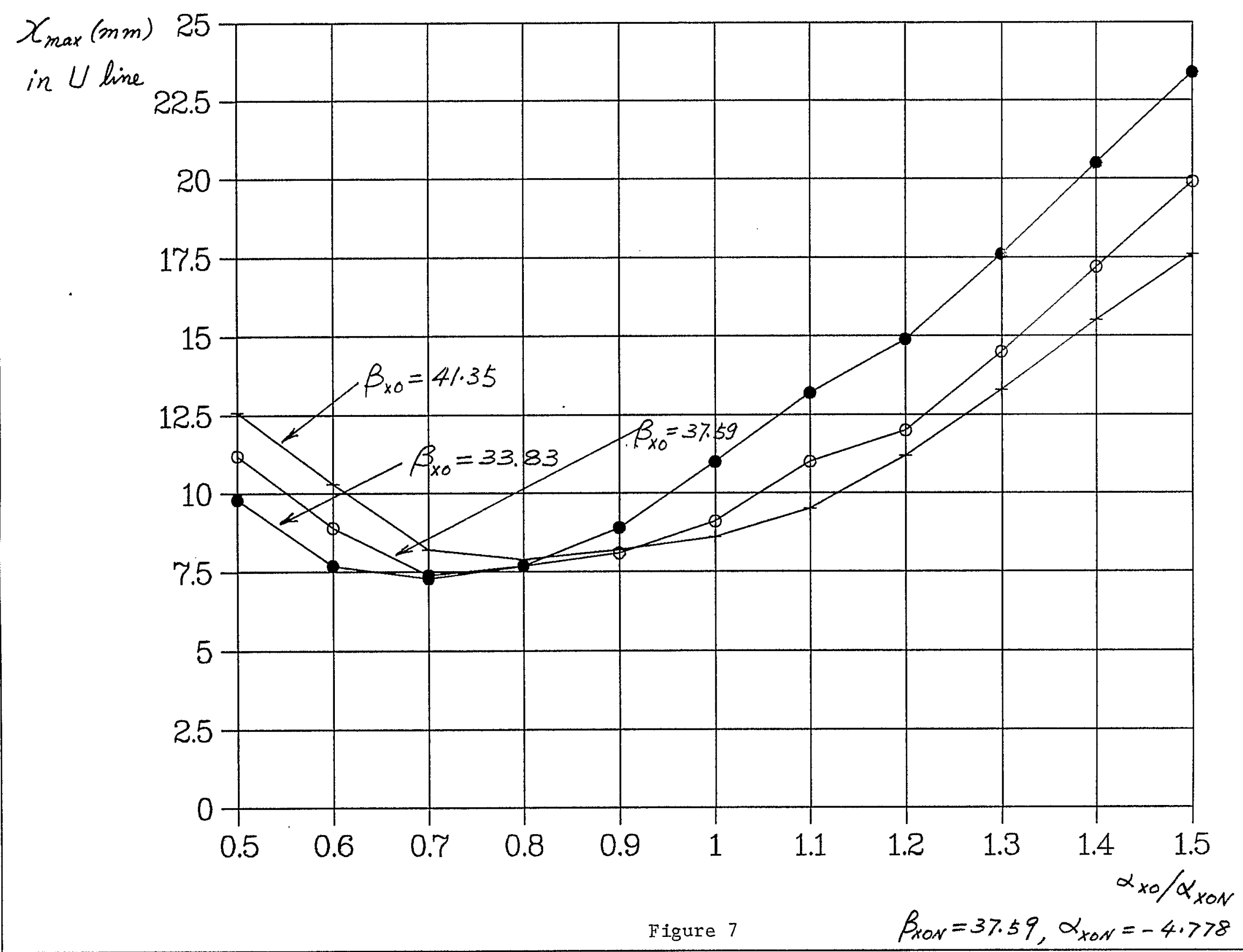




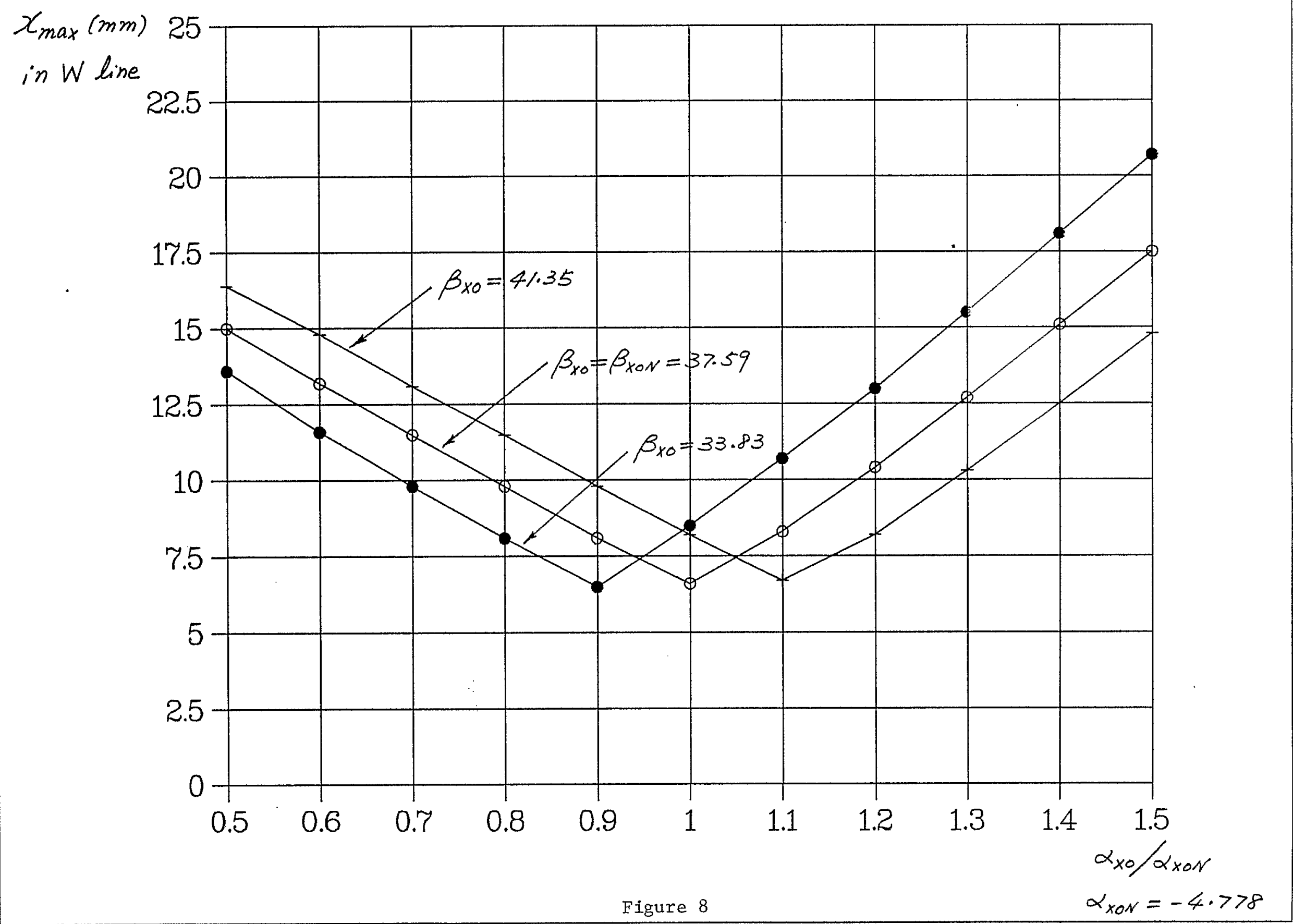




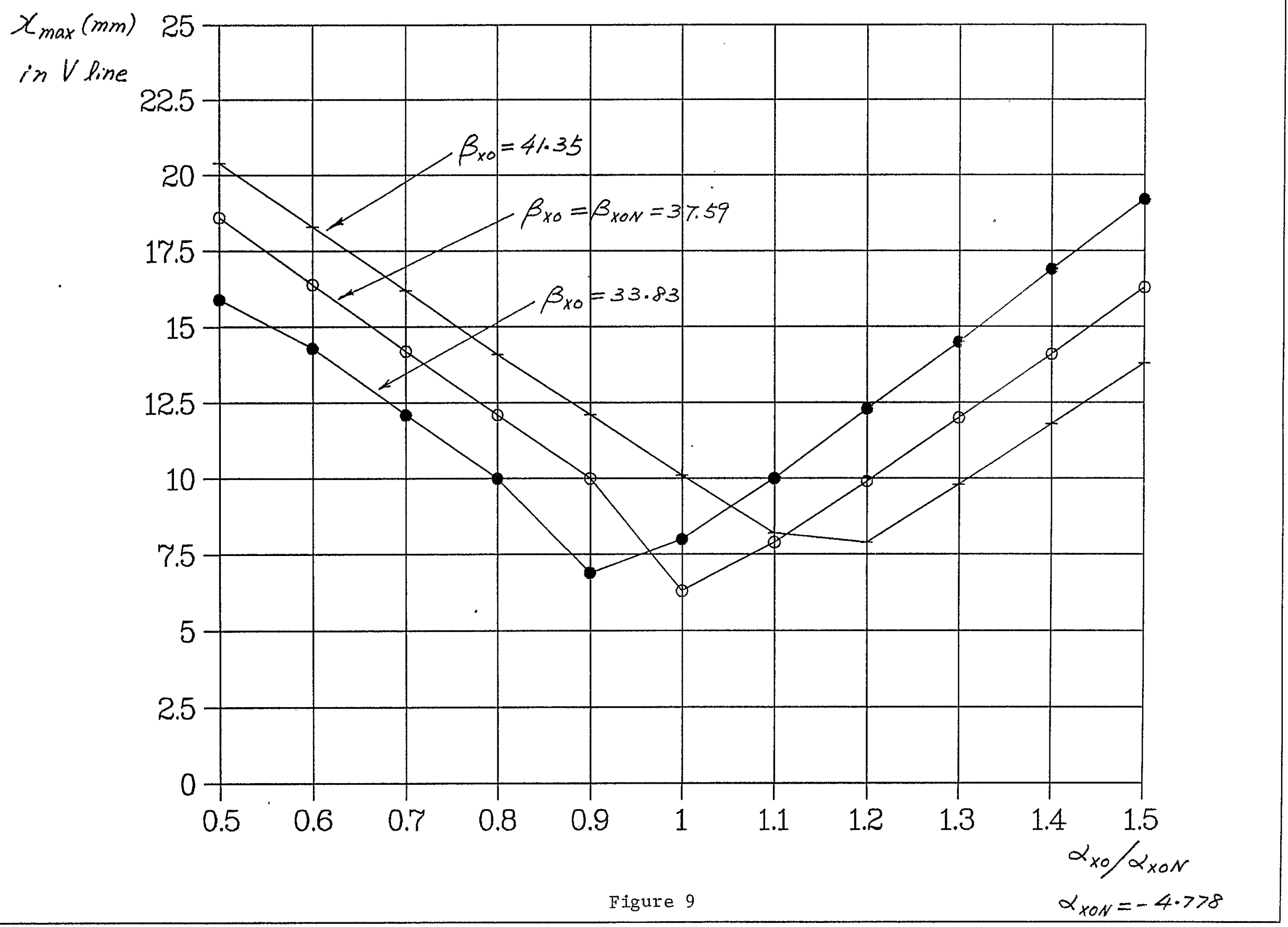




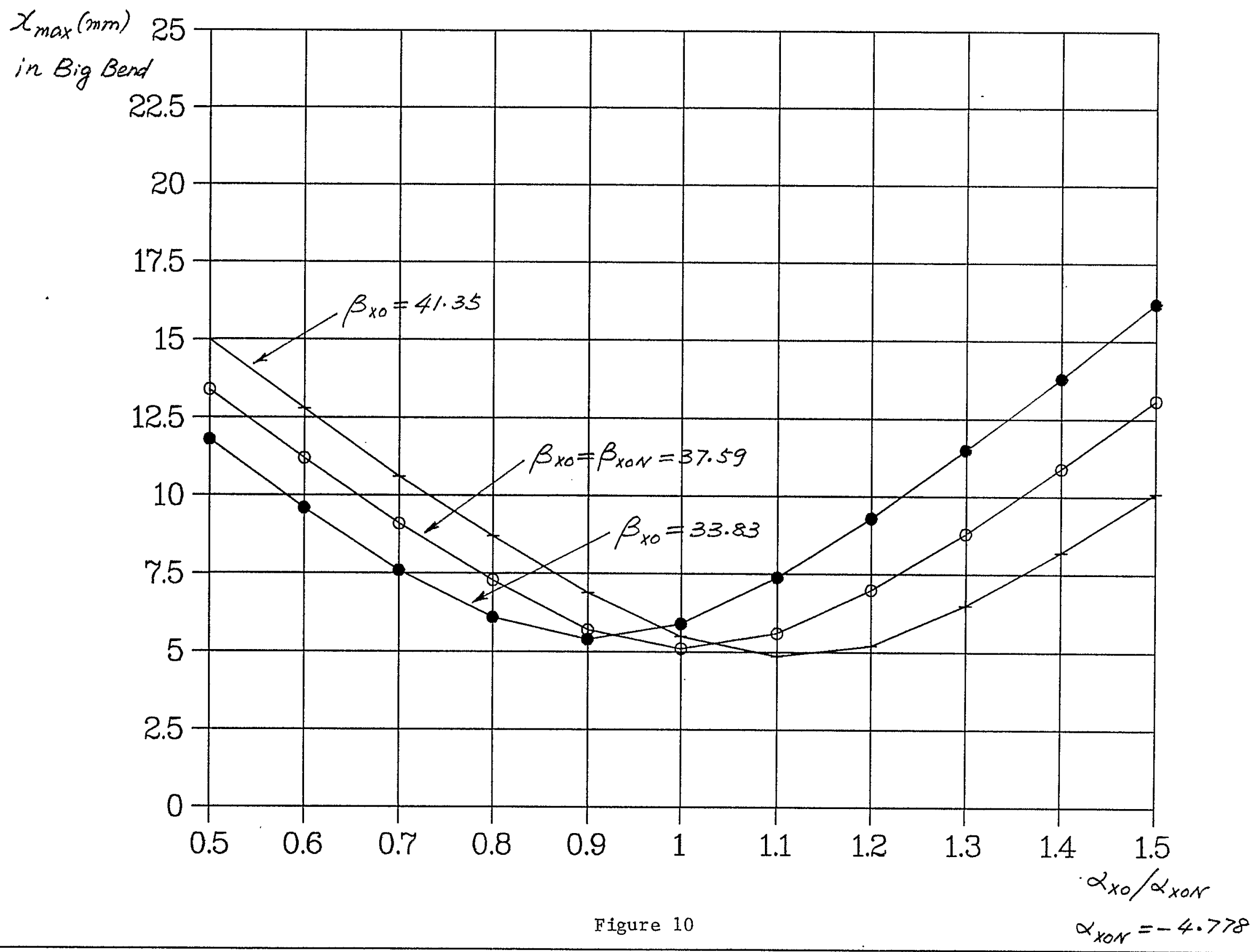




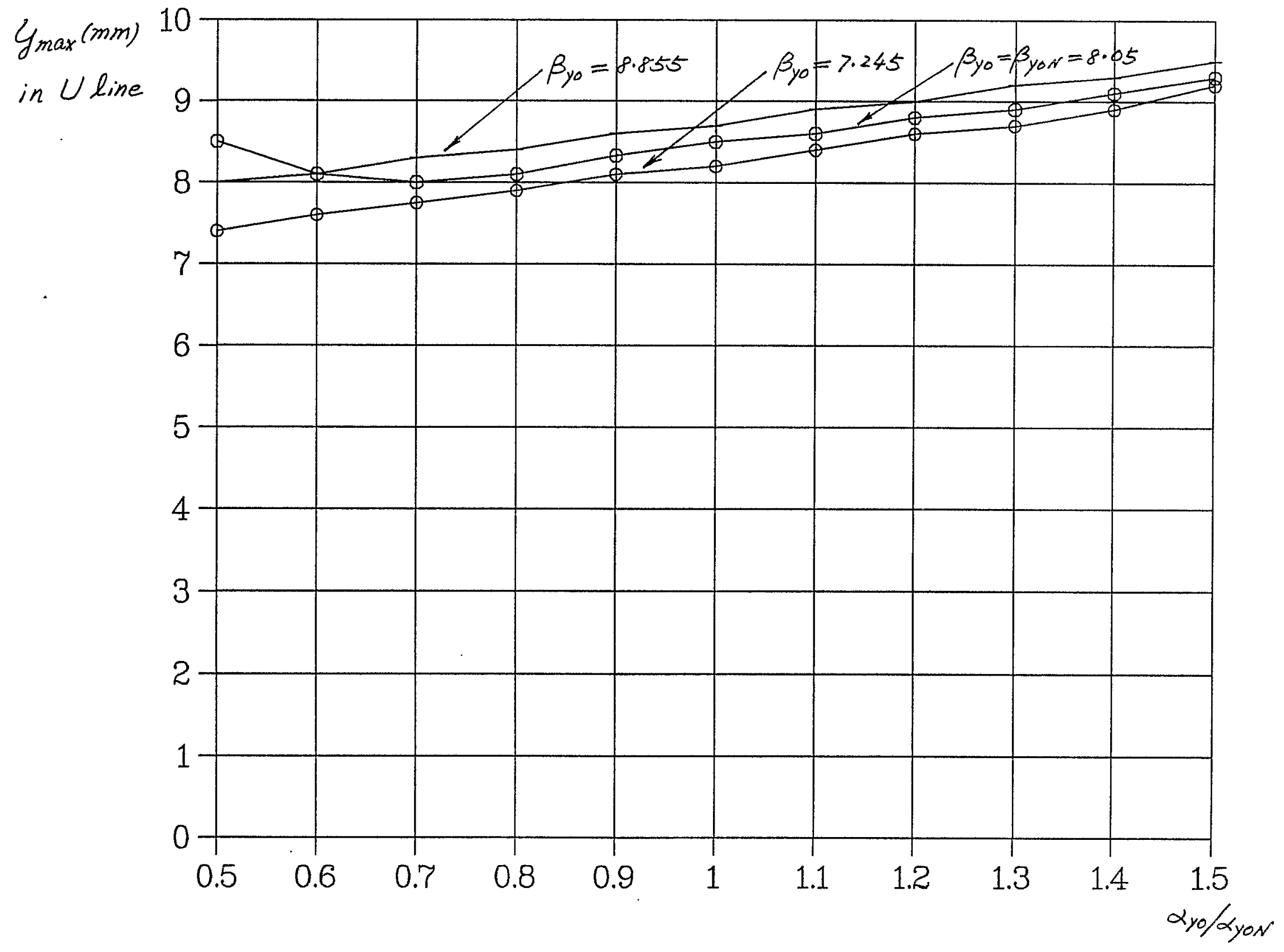




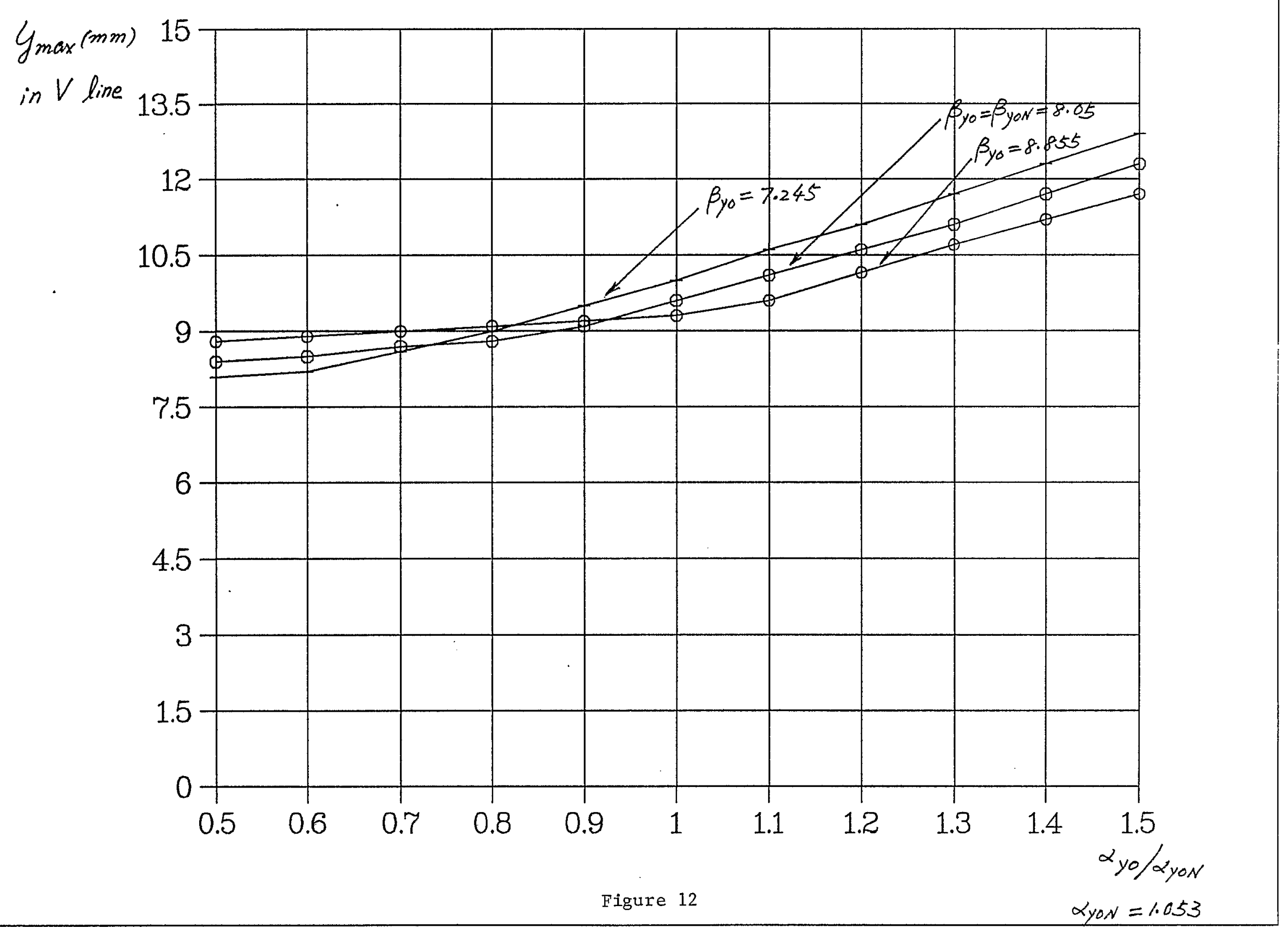




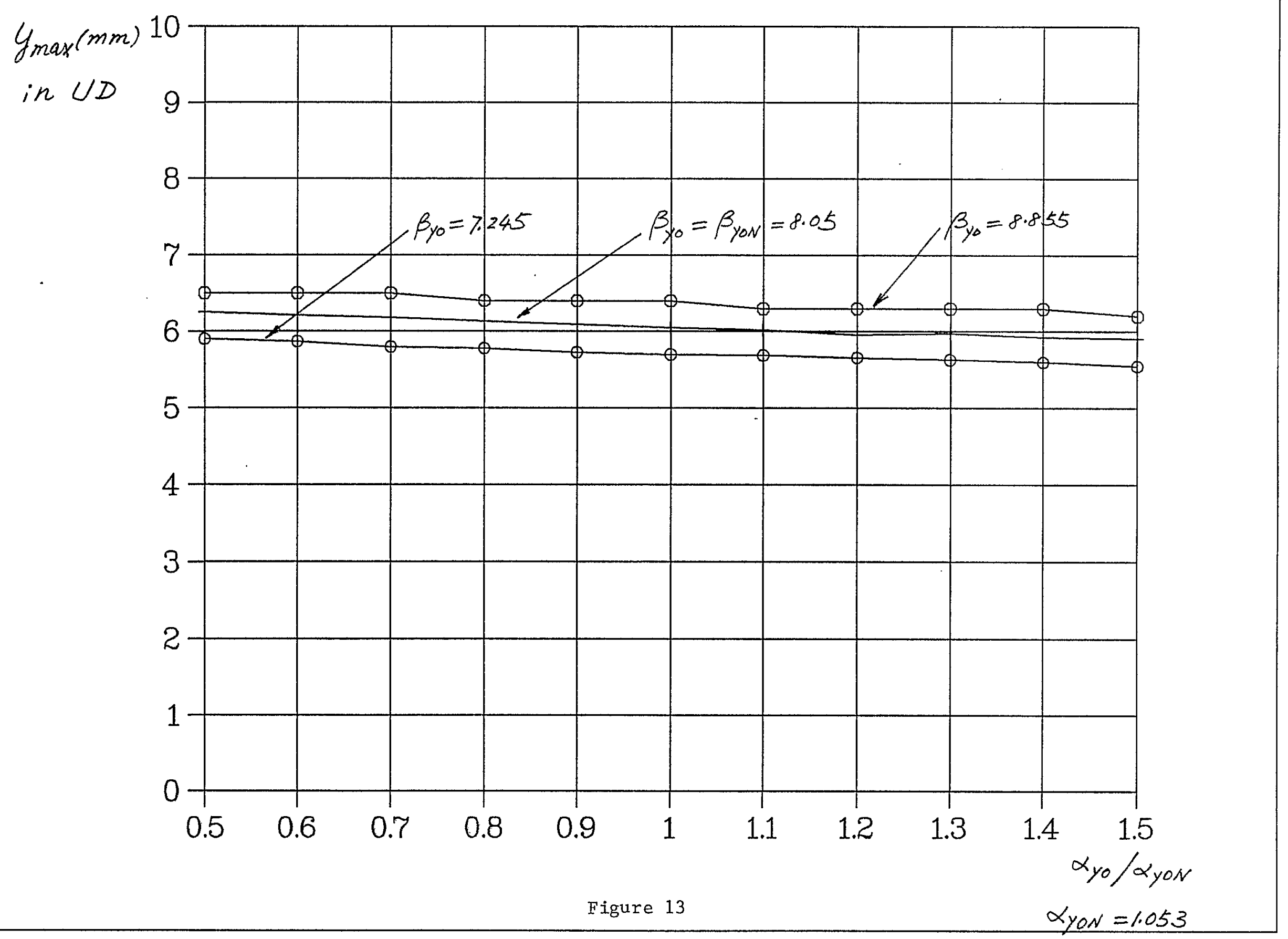




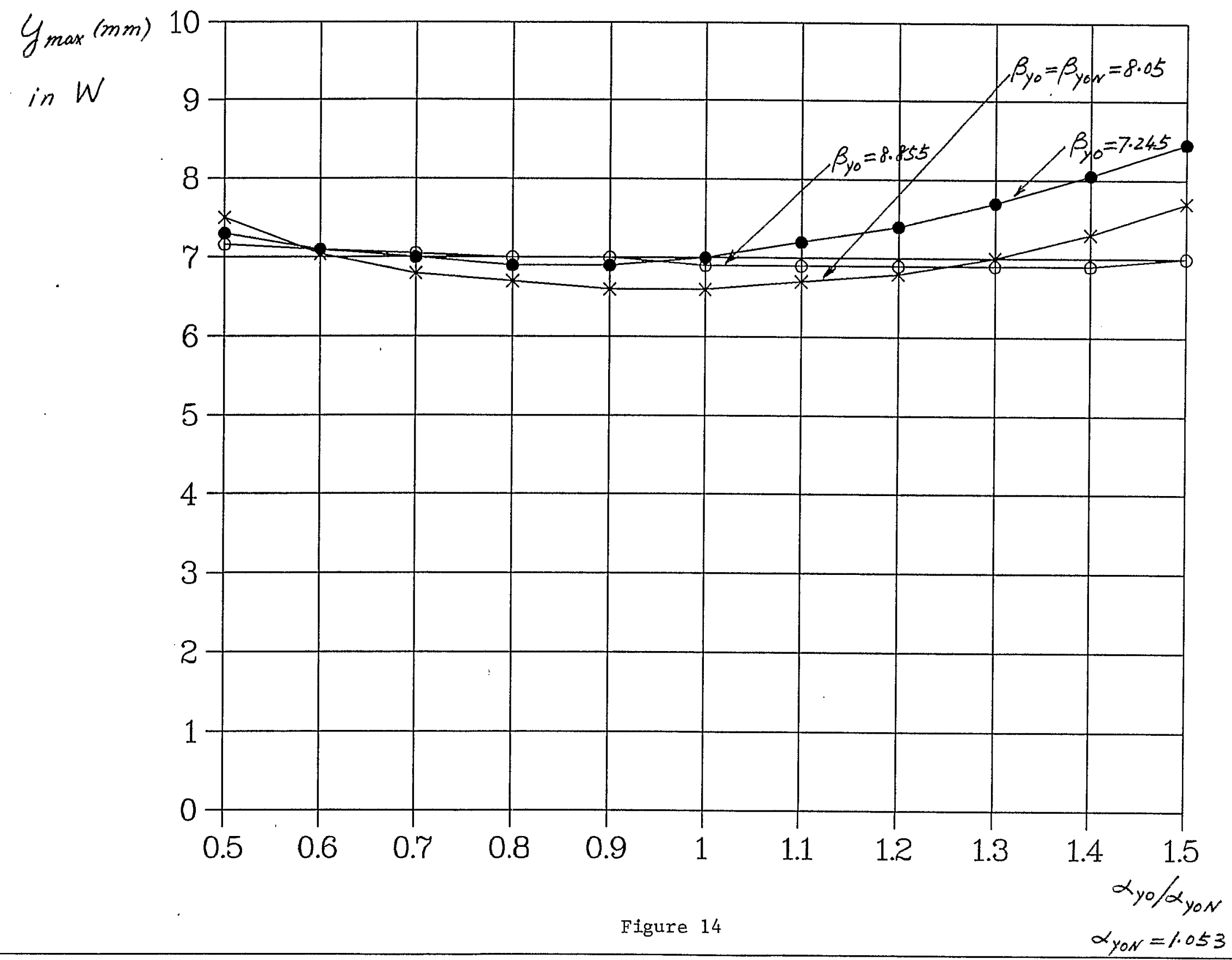




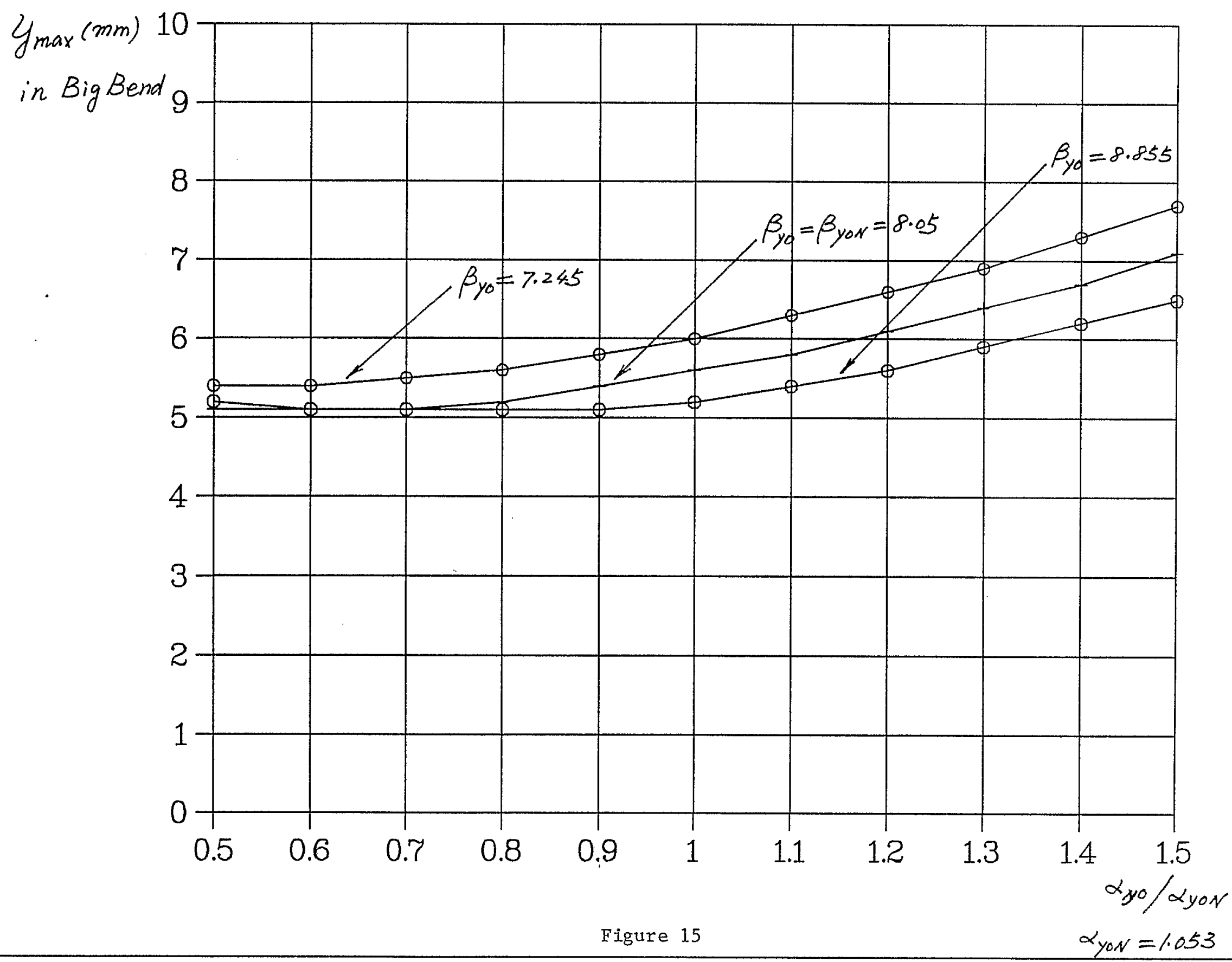




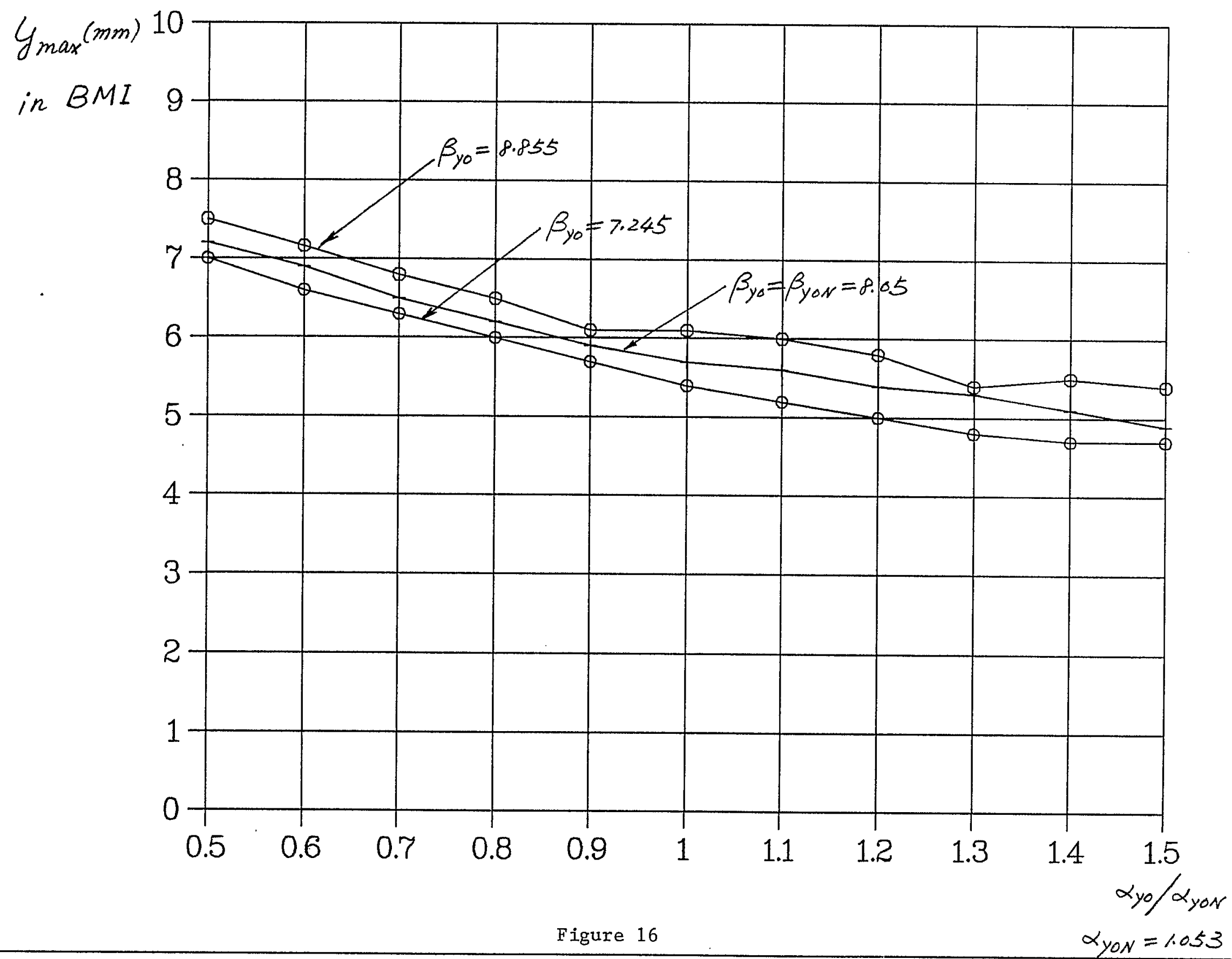




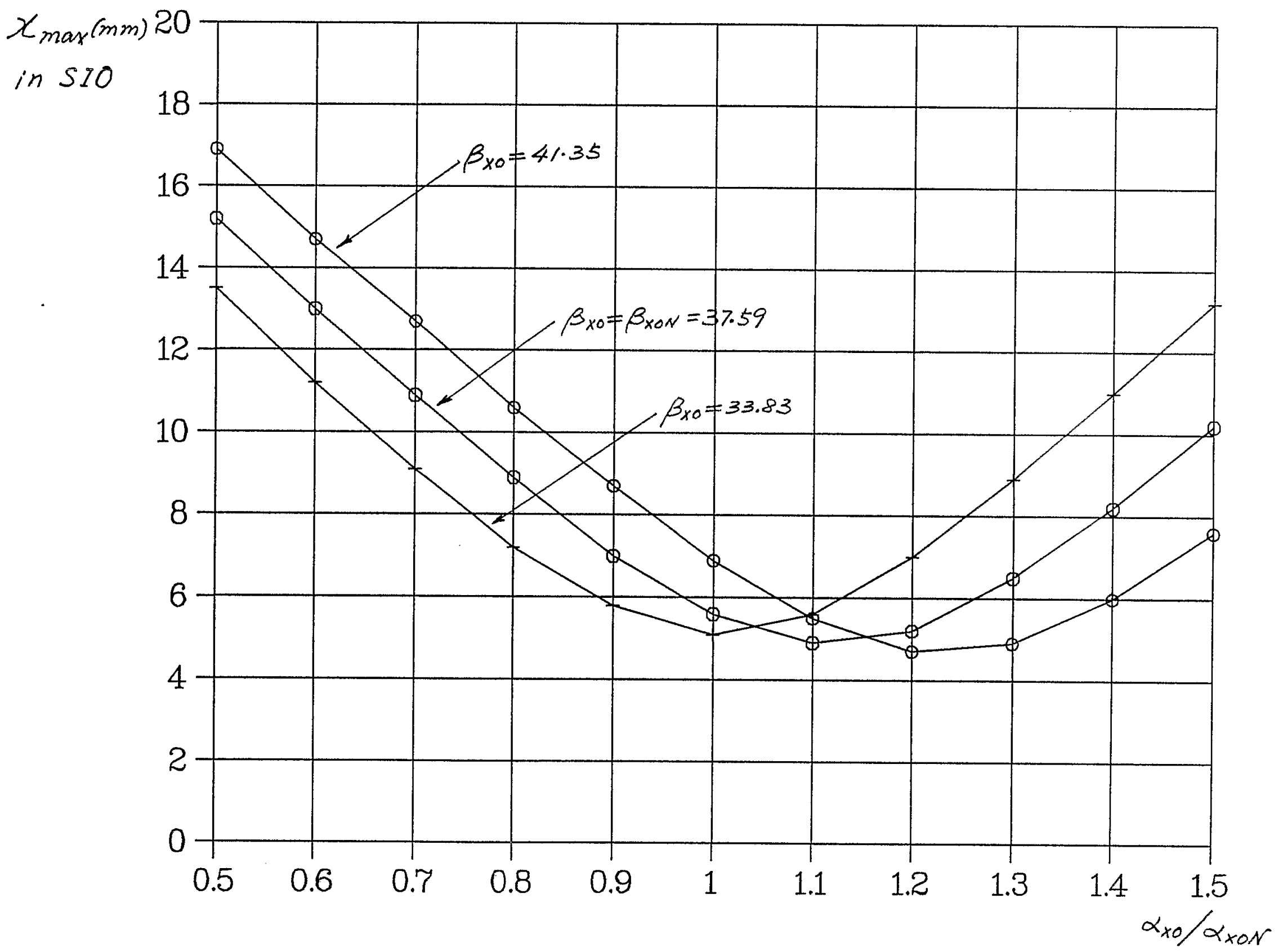

Figure 17

$\alpha_{\text {XON }}=-4.778$ 


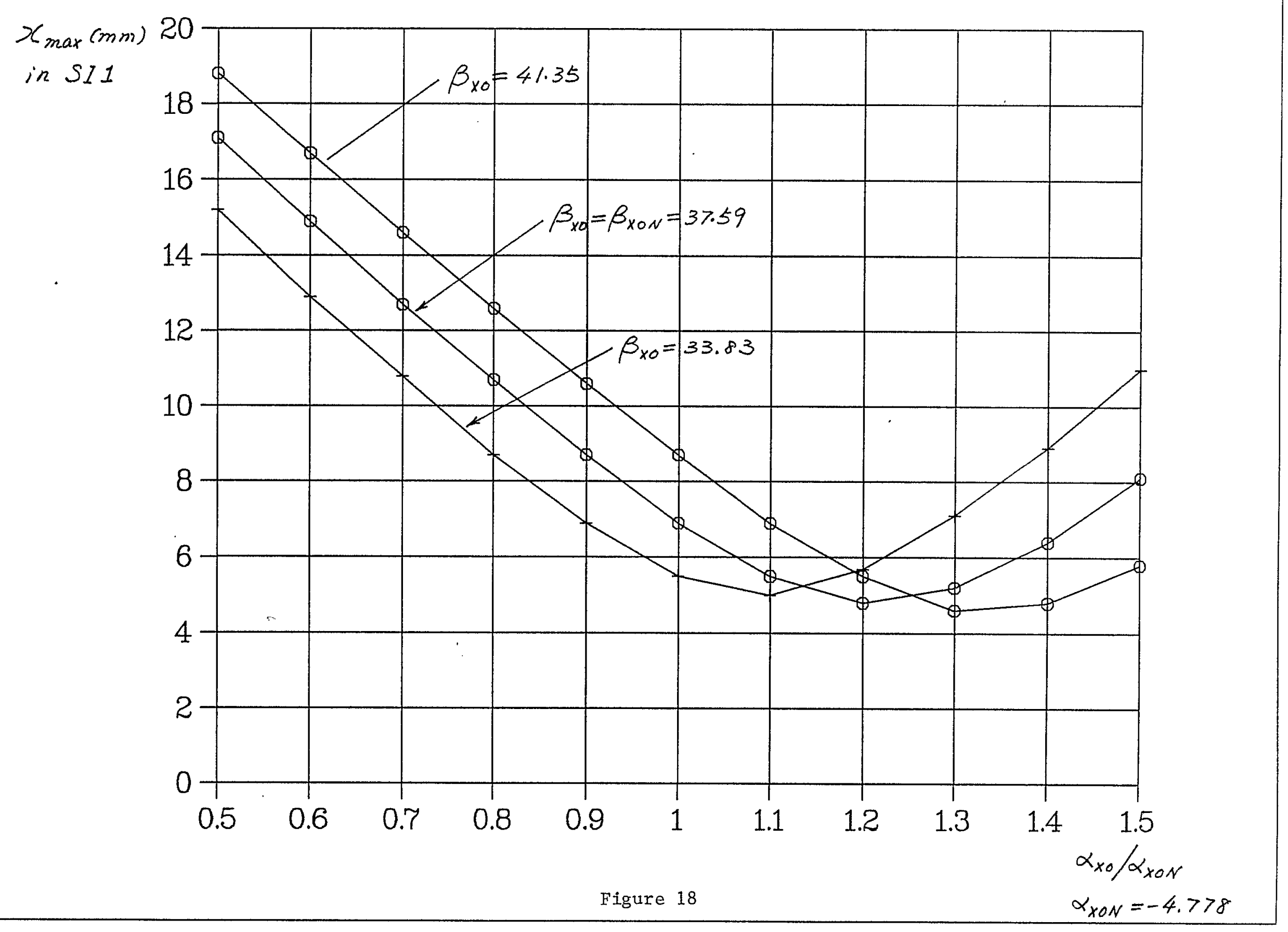




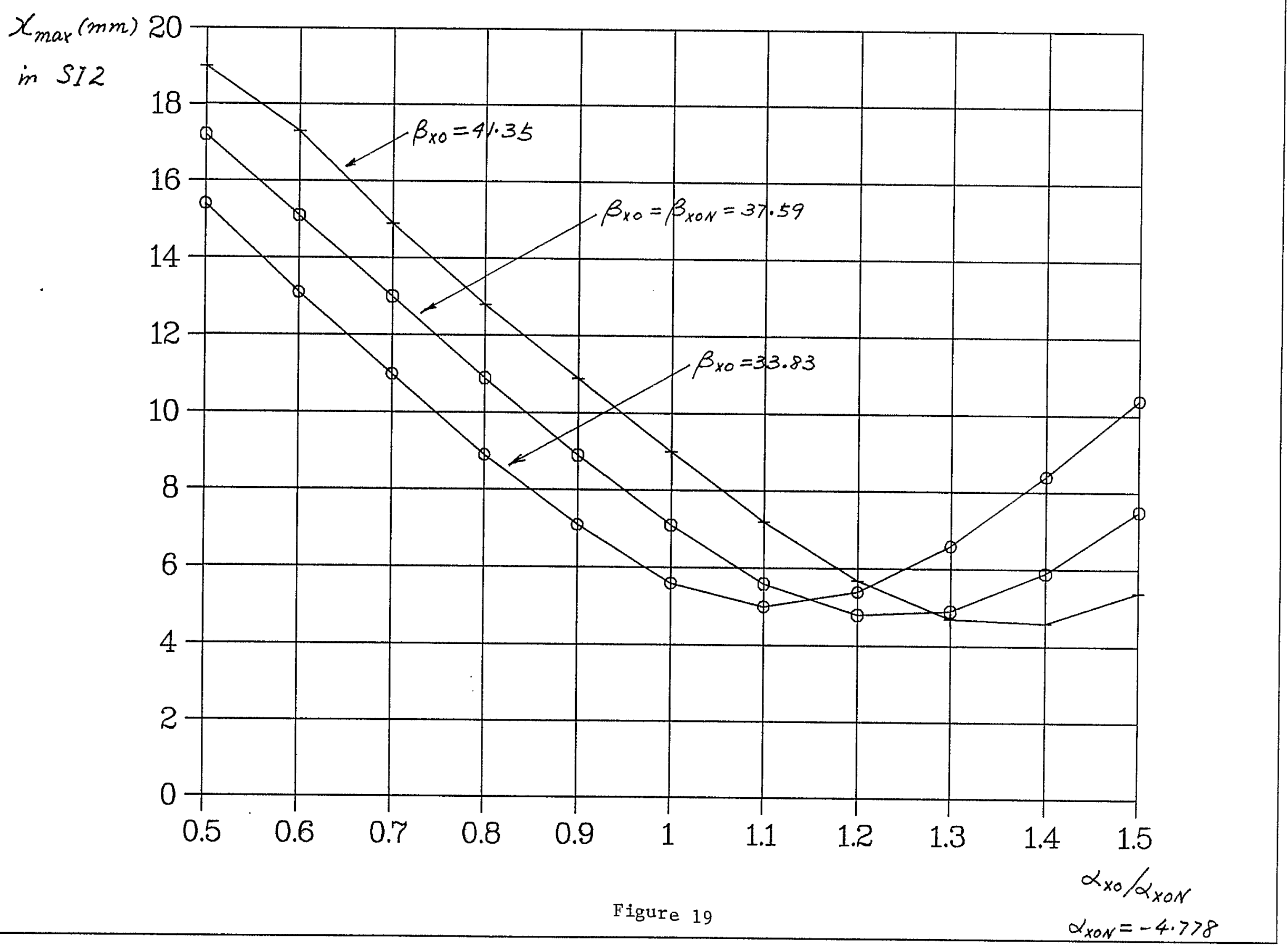




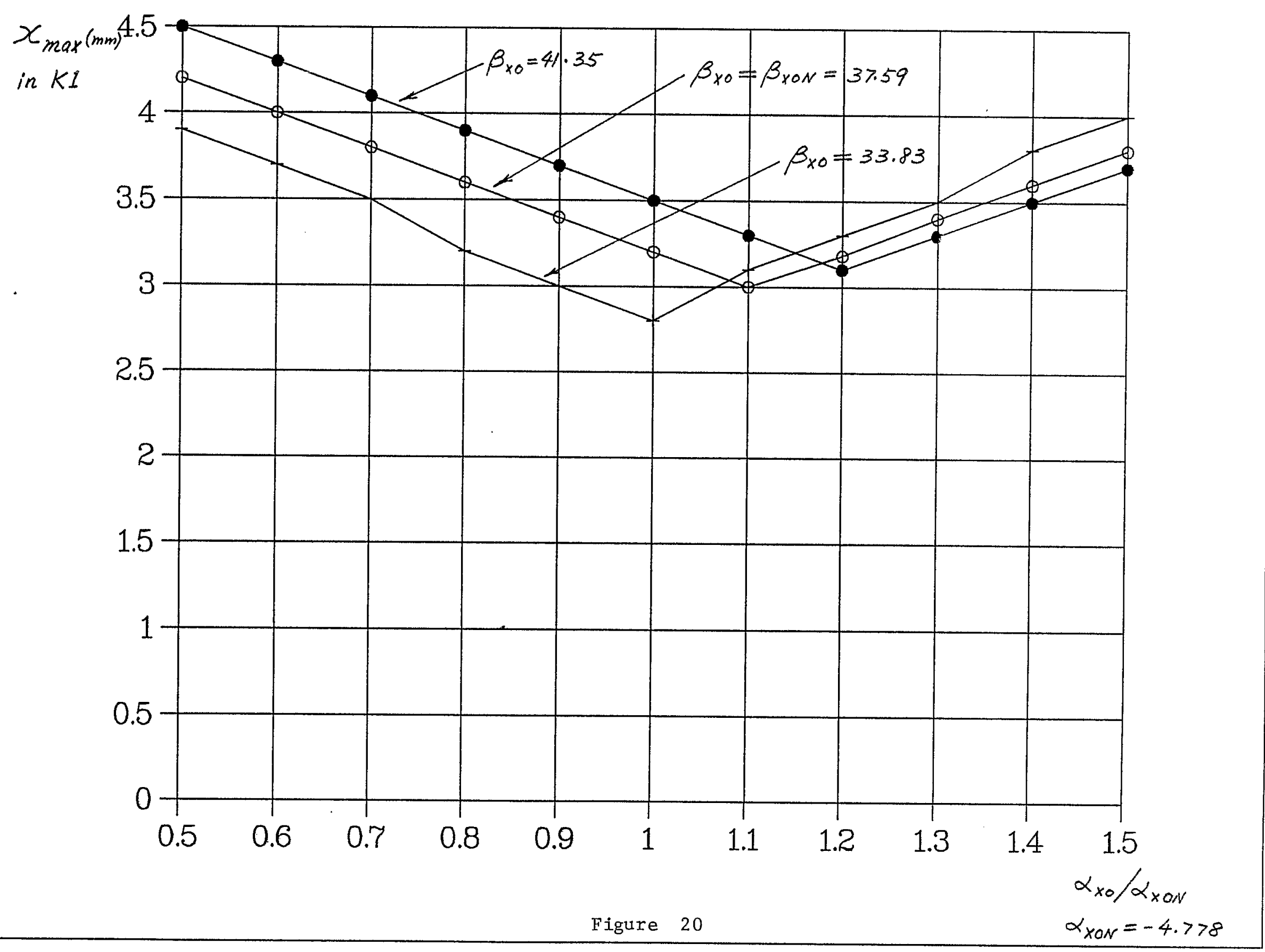




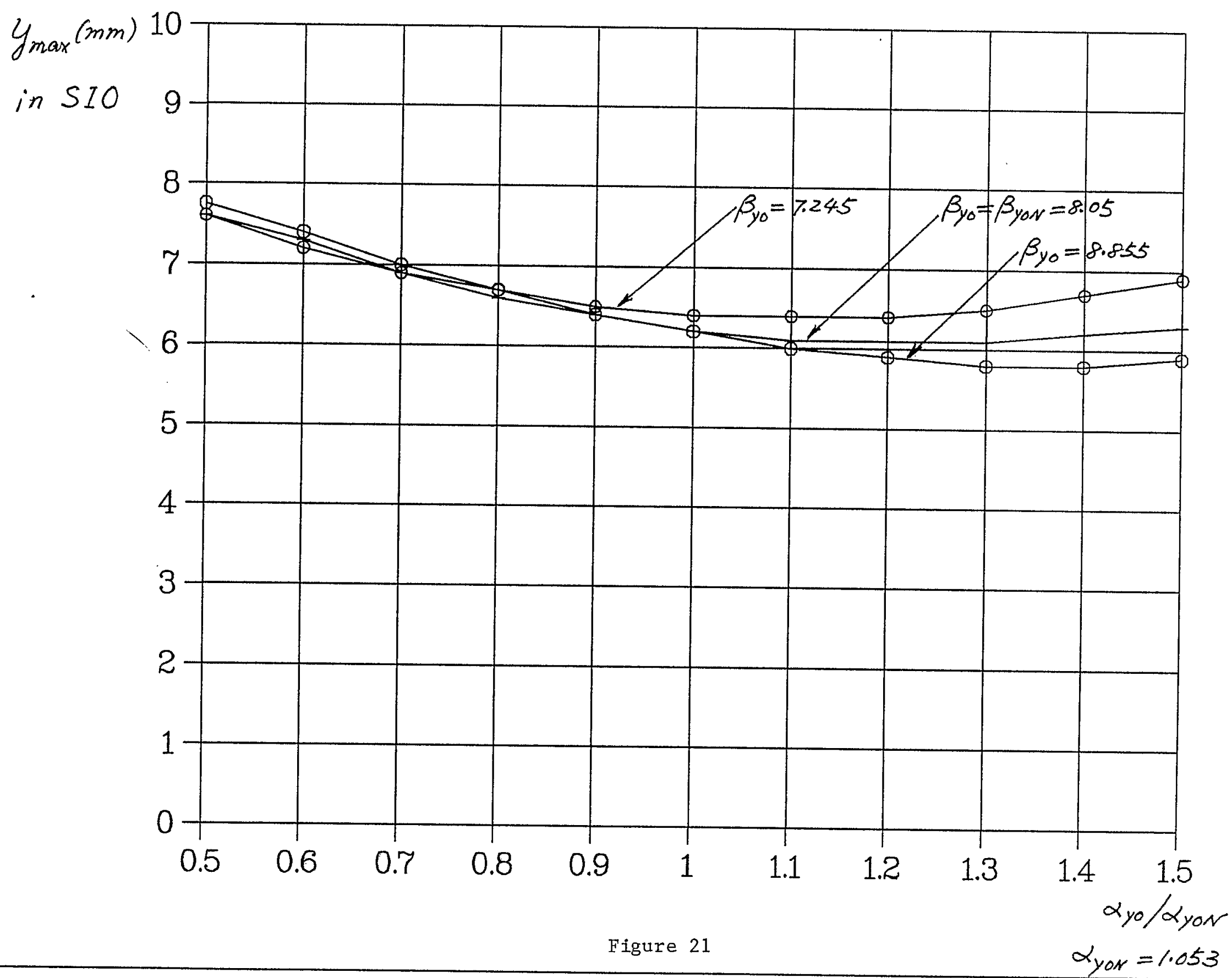




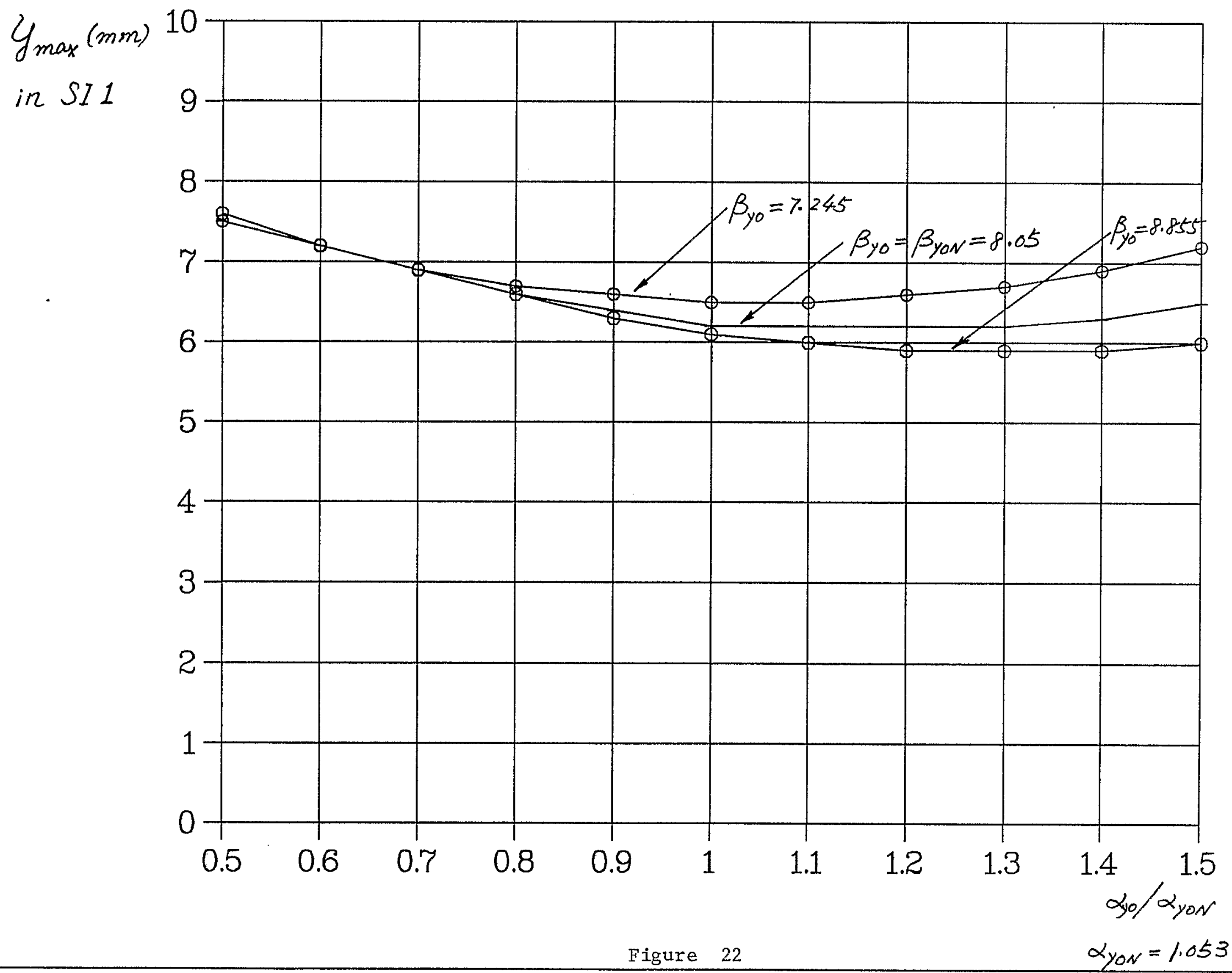




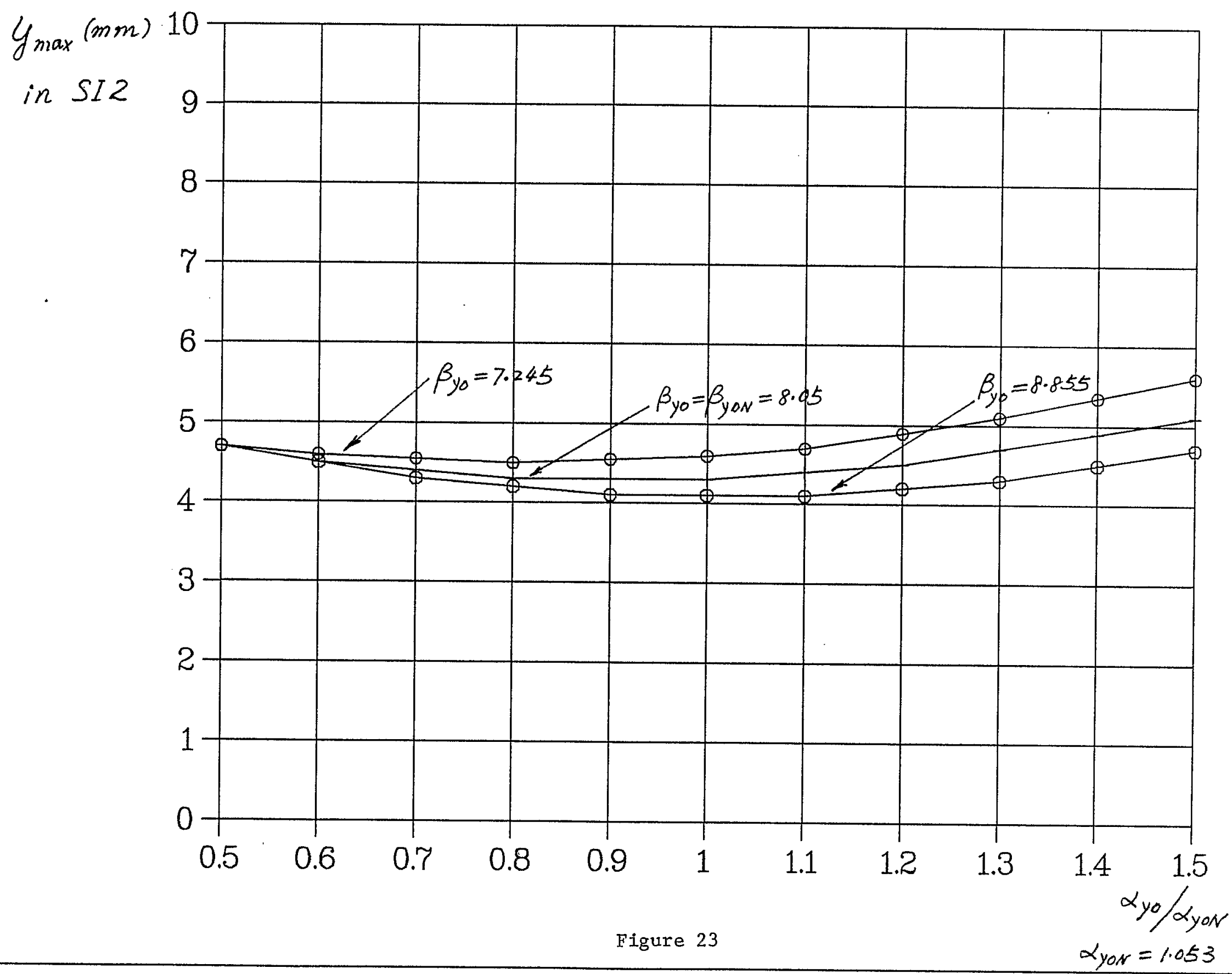




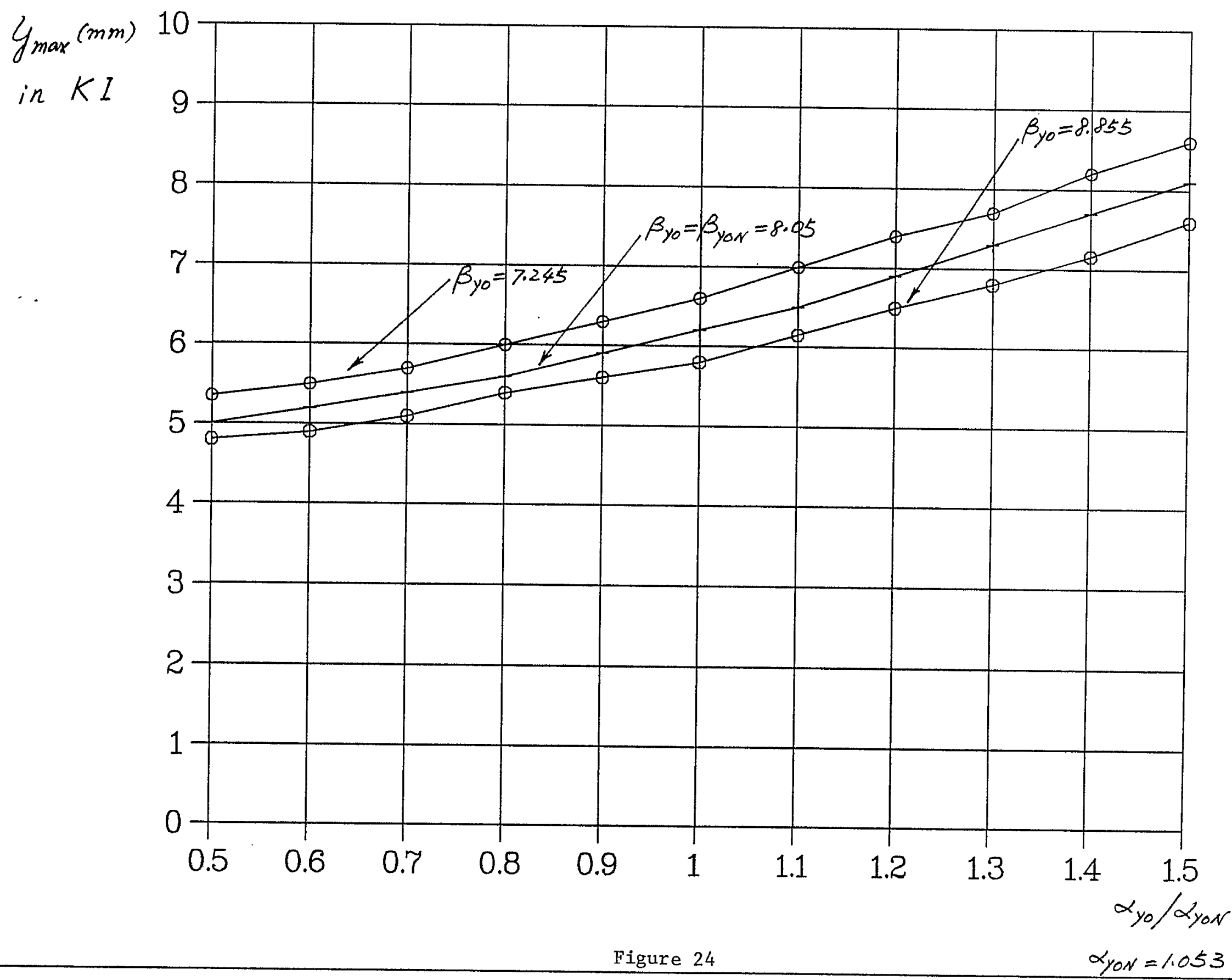

